\title{
A Brief Summary of VSPEC ${ }^{\star}$
}

\author{
Perry Alexander ${ }^{1}$, Murali Rangarajan ${ }^{1}$, and Phillip Baraona ${ }^{2}$ \\ 1 Department of Electrical \& Computer Engineering \\ and Computer Science \\ PO Box 210030 \\ The University of Cincinnati \\ Cincinnati, $\mathrm{OH}$ \\ \{alex,rmurali\}@ececs.uc.edu \\ 2 Chrysalis Symbolic Design, Inc. \\ 101 Billerica Ave \\ 5 Billerica Park \\ Billerica, MA 01862 \\ phil@chrysalis.com
}

\begin{abstract}
This paper provides an overview of the VSPEC behavioral interface specification language for VHDL. Although operational specification language such as VHDL provide exceptional specification capabilities, at the systems requirements level the operational style is a hindrance. VSPEC provides VHDL users with a declarative mechanism for defining functional requirements and performance constraints. In the tradition of behavioral interface specification languages, VSPEC adds clauses to the VHDL entity construct allowing axiomatic specification of functional requirements. Because system constraints play an ever increasing role in systems design, VSPEC also provides performance constraint specification capability. This paper presents the basics of VSPEC, its semantics, semantic analysis, and briefly describes current and future applications.
\end{abstract}

Keywords: systems level design, notations, languages, VHDL, Larch

\section{Introduction}

Requirements analysis is a critical activity in any systems design process. However, it is poorly supported by tools and languages. Although operational, simulation centered, hardware description languages such as VHDL [1 provide excellent support for design, they are less appropriate for requirements analysis. The operational style tends to introduce implementation bias into requirements. Furthermore, simulation-based analysis is not always appropriate for evaluating highly declarative, frequently incomplete requirements. To address such problems, VSPEC [2, 3, 4, 5] augments VHDL to provide a declarative requirements specification capability that support rigorous, formal analysis.

\footnotetext{
* Support for this work was provided in part by the Advanced Research Projects Agency and monitored by Wright Labs under the RASSP Technology Program, contract number F33615-93-C-1316 and by Air Force MANTECH, contract number F33615-93-C-4303
} 
VSPEC is a Larch interface language [6] 7] for VHDL. The Larch family of specification languages supports a two-tiered, model-based approach to specifying software. A Larch specification is written in two languages: a Larch Interface Language (LIL) and the Larch Shared Language (LSL). Larch Interface Language definitions specify the inputs and outputs of a program component and the component's observable behavior. Typically, input and output parameters are defined in the host programming language. Then, first order predicates define component behavior using a traditional axiomatic style. Larch Interface Languages exist for a variety of programming languages, including $\mathrm{C}$ [8] 9], $\mathrm{C}++[10$ ] and Ada 11].

LSL is a formal algebraic language that defines the underlying sorts and operators used in interface language definitions. As the name implies, LSL is common among all Larch Interface Languages. Specifiers use LSL to define reusable domain theories for specification activities and to define semantics for interface languages.

VSPEC describes the requirements of a digital system using the canonical Larch approach. Each VHDL entity is annotated with a pre- and post-condition to indicate the component's functional requirements. The operators used in a VSPEC description are defined with LSL. VSPEC also allows a designer to describe non-functional requirements and the internal state of a device. VSPEC semantics is defined by providing a translation of VSPEC language constructs and VHDL types into LSL enabling formal verification using Larch tools.

VSPEC-annotated components can be connected together to form an abstract architecture. An abstract architecture is an inter-connected collection of components where the requirements of each component are specified without defining their implementation. This describes a class of solutions with a common structure. A standard VHDL structural architecture referencing VSPEC annotated entities defines an abstract architecture. The VHDL architecture indicates interconnection in the traditional manner, but the requirements of each component are defined instead of their implementations.

Abstract architectures specified with VSPEC present a problem that other Larch interface languages do not have to address: when is a component in an abstract architecture active? In traditional sequential programming languages, a language construct executes after the construct immediately preceding it terminates. For correct execution, a construct's pre-condition must be satisfied when the preceding construct terminates. In a VSPEC abstract architecture, each of the components behave as independent processes. There is no predefined execution order so there is no means of determining when a component's pre-condition should hold. VSPEC solves this problem by allowing a user to define an activation condition for a component. The activation condition defines what causes the component to begin processing its inputs. When the component state changes to one that satisfies the activation condition, the pre-condition must hold and the component performs its specified transformation.

This paper describes the semantics of VSPEC, concentrating on the language's facilities for describing abstract architectures. The opening section provides a brief summary of the VSPEC language. Section 3 describes VSPEC abstract ar- 
chitectures, including a definition of the VSPEC state model and a description of how a process algebra (CSP) 12 is used to provide a semantics for the VSPEC activation condition. Section 5 discusses how these semantics can be used verify that an abstract architecture satisfies the specification of the entity. Finally, the paper concludes with a discussion of VSPEC applications and some related work.

\section{The VSPEC Language}

VSPEC's declarative specification style complements the traditional VHDL operational style by providing a requirements specification capability. As a requirements specification language, VSPEC is used very early in the design process to describe "what" a system should do. The operational style of VHDL makes VHDL alone ill-suited for requirements specification. It forces a designer to describe a system by defining a specific design artifact that describes "how" the system behaves. When attempting to use VHDL as a requirements specification language, this forces a designer to deal with unnecessary detail at a very early point in the design process. In contrast to VHDL's operational style, vSPEC allows a designer to declaratively describe a component. Together, VSPEC and VHDL support modeling from requirements acquisition through verification and synthesis.

As a working example, a VSPEC description of a sorting component is shown in Figure 1 Three basic clauses define functional requirements for an entity: (i) the requires clause defines the component precondition; (ii) the ensures clause defines the component postcondition; and (iii) the sensitive to clause defines the component activation condition. Effectively, the requires and ensures clauses define component function while the sensitive to clause defines component control.

The requires and ensures clauses are used to define an axiomatic relationship between current and the next state. Specifically, they specify the pre- and post-conditions of the component. Any component that makes the postcondition true in the next state given that the precondition is true in the current state is a valid implementation of these requirements. More precisely, given a component with requires clause $I(S t)$ and ensures clause $O\left(S t, S t^{\prime}\right.$ post $\left.)\right)^{1}, f(S t)$ is a correct implementation of the requirements if the following condition holds:

$$
\forall s \cdot I(S t) \Rightarrow O(S t, f(S t))
$$

The sensitive to clause plays the same role in a VSPEC definition that sensitivity lists and wait statements play in a VHDL description. It defines when a component is active. The sensitive to clause for sort in Figure 1 states that the entity activates (and sorts its input) whenever the input changes. The sensitive to clause contains a predicate indicating when an entity should begin

${ }^{1}$ The $S t^{\prime}$ post notation references the value of $S t$ in the state after the transformation described by the entity is performed. This is analogous to the variable notation of LCL 89 


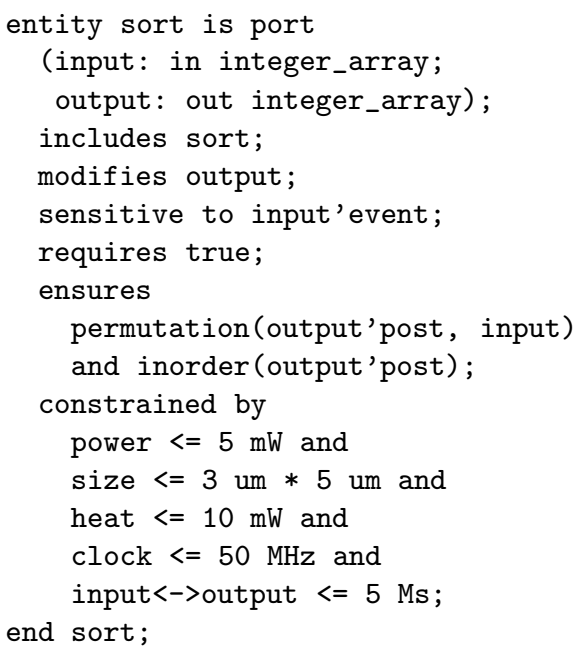

Fig. 1. VSPEC description of a sorting component.

executing and the next section contains a more precise definition of the meaning of the sensitive to predicate.

In the example specification from Figure 1 the sORT component is defined to operate correctly in any initial state whenever its input changes and produce an output that is ordered and a permutation of the input. The requires clause defines a precondition of true. As true holds in any state, the component must execute starting in any state. The ensures clause defines a postcondition of permutation(input, output'post) and ordered(output'post) requiring that after execution the output should be an ordered permutation of the input. Note that ordered and permutation are defined in the included trait sort. The sensitive to clause defines an activation condition of input' event. Event is a predefined VSPEC predicate that is true whenever its associated signal changed values in the previous state change.

In addition to allowing a designer to describe functional requirements, VSPEC also allows specification of performance constraints. The VSPEC constrained by clause is used for this purpose. As shown in Figure 1 this clause defines relations over constraint variables. Currently, the defined constraint variables include power consumption, layout area (expressed as a bounding box), heat dissipation, clock speed and pin to pin timing. Constraint theories written in LSL define each constraint type. Users may define their own constraints and theories if desired 2

The state clause contains a list of variable declarations that define the internal state of a component. These variables maintain state information that is not

${ }^{2}$ VSPEC constraint specification is not presented in detail in this paper. For further exposition, please see the Language Reference Manual [13]. 


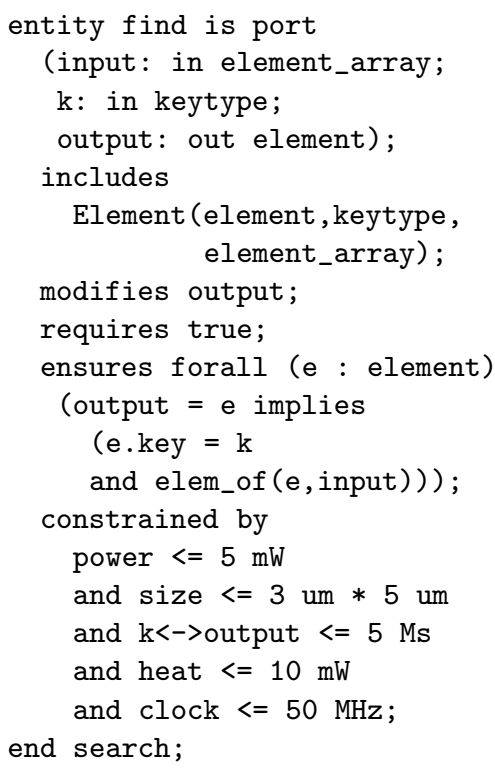

(a.)

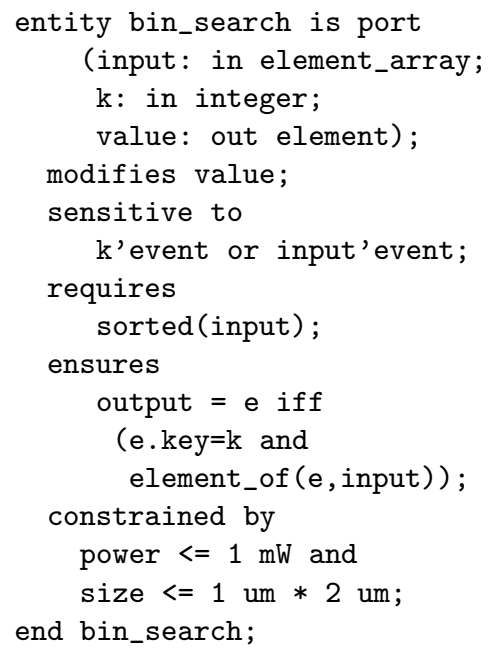

(b.)

Fig. 2. VSPEC descriptions of find and binary search components.

visible outside the component. A state clause is not needed in the specification of a sorting component in Figure 1 as no internal state is stored.

The modifies clause lists variables, ports and signals whose values may be changed by the entity. Most other Larch interface languages contain a modifies clause, and the definition of VSPEC modifies clause is very similar to the definitions found in these languages [8, 9, 10,

The includes clause is used to include Larch Shared Language definitions in a VSPEC description. The sorts and operators defined in the LSL trait named by the includes clause can be used in the VSPEC definition.

\section{Abstract Architectures}

VHDL structural architectures composed only of VSPEC annotated components specify abstract architectures. The VHDL architecture syntax remains unchanged indicating component instantiation and connections. However, the configuration does not assign an entity/architecture pair to each component instance in the architecture. Instead, the configuration defines that each component references an entity with an architecture called VSPEC. This signifies that at the current point in the design, the requirements of this component are known (via the VSPEC description) but no implementation has been defined.

Consider the VSPEC description of a find component shown in Figure $2 \mathrm{a}$. The output of find is the element from the input array with the same key as 
clause contains a predicate called the activation condition that indicates when an entity should begin executing. Effectively, this activation condition defines when a VSPEC annotated entity's precondition must hold. When the sensitive to predicate is true, the pre-condition must hold and the implementation must satisfy the post-condition. When the sensitive to predicate is false, the entity makes no contribution to the state of the system. In the find example, both components activate when any of their input signals change.

Formally, the contribution of the sensitive to clause to the transformation specified by VSPEC is easily represented using a traditional process algebra such as CSP [12]. Components become processes and events are defined as the states the entity enters. Thus, any VSPEC component can be described by a process that consumes states and generates a process in a new state. To define such state changes, a component state is defined along with a means for combining component states into an architecture state.

The formal vSPEC model of the state of a component is based on Chalin's state model [8] Chapter 6] for LCL. This model partitions the computational state of an LCL description into an environment and a store [14. The environment maps (variable) identifiers into objects and the store binds objects to the values they contain:

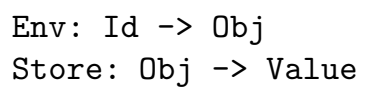

Separating the environment and the store is common among formal models of programming language semantics. In a language such as LCL, one of the motivating factors for this is to allow multiple names for the same element of memory. For example, two $\mathrm{C}$ pointers can obviously reference the same memory location. The program state model above represents this situation by mapping each of these pointers to the same object in the Env map.

This partitioning of the state of a component is used in the VSPEC semantics to model component communication. For a single vsPEC-specified component, Env contains a map from each port and state variable in the VSPEC description to an object. Store maps each of these objects to their current value. We call this the abstract state of the VSPEC component. When VSPEC components are connected together to form an abstract architecture, the elements of Env and Store are slightly different. The Store contains objects for each port in the architecture's entity, for each signal in the architecture and for the state variables of each component in the architecture. The Env maps each of these three types of elements to the proper object, but it also maps the ports of each architecture component to the object that represents the architecture signal the port is connected to. We call the state model of an abstract architecture the concrete state of the component.

In the simple two component example of Figure 4 the abstract state of system, $\mathrm{A}$ and $\mathrm{B}$ are: 

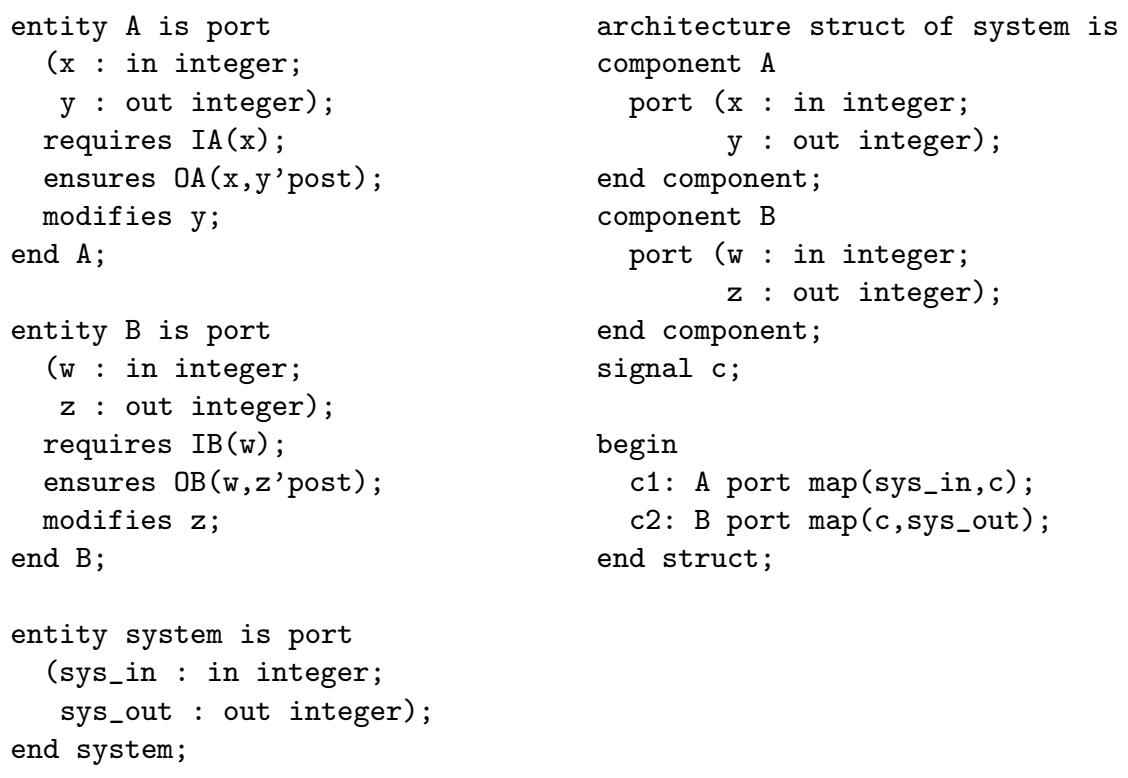

Fig. 4. Example of two entities connected serially.

$$
\begin{aligned}
\text { Env }_{\text {system }_{\text {stem }}} & =\left\{s y s_{\_} i n \mapsto o b j_{\text {sys_in }}, s y s_{\_} \text {out } \mapsto o b j_{\text {sys_out }}\right\} \\
\text { Store }_{\text {system }} & =\left\{o b j_{\text {sys_in }} \mapsto v_{\text {sys_in }}, o b j_{\text {sys_out }} \mapsto v_{\text {sys_out }}\right\} \\
\text { Env }_{A} & =\left\{x \mapsto o b j_{x}, y \mapsto o b j_{y}\right\} \\
\text { Store }_{A} & =\left\{o b j_{x} \mapsto v_{x}, o b j_{y} \mapsto v_{y}\right\} \\
\text { Env }_{B} & =\left\{w \mapsto o b j_{w}, z \mapsto o b j_{z}\right\} \\
\text { Store }_{B} & =\left\{o b j_{w} \mapsto v_{w}, o b j_{z} \mapsto v_{z}\right\}
\end{aligned}
$$

The concrete state of the struct architecture is:

$$
\begin{aligned}
& \text { Env }_{\text {struct }} \text { system }=\left\{s y s_{-} i n \mapsto o b j_{\text {sys_in }}, s y s_{-} o u t \mapsto o b j_{s y s_{-} o u t}, c \mapsto o b j_{c}\right. \text {, } \\
& \left.x \mapsto o b j_{\text {sys_in }}, y \mapsto o b j_{c}, w \mapsto o b j_{c}, z \mapsto o b j_{\text {sys_out }}\right\} \\
& \text { Store }_{\text {struct }} \text { system }=\left\{o b j_{\text {sys_in }} \mapsto v_{\text {sys_in }}, o b j_{\text {sys_out }} \mapsto v_{\text {sys_out }}, o b j_{c} \mapsto v_{c}\right\}
\end{aligned}
$$

Notice that $\mathrm{x}, \mathrm{y}, \mathrm{w}$ and $\mathrm{z}$ now map to the objects that contain the values of the signals that these component ports are connected to in the architecture.

Using a component's state, its semantics are defined using a CSP process and its requires and ensures clauses. Let $f(S t)$ be a function between two states of entity $C$ that implements the requirements specified in $C$ 's requires and ensures clauses (i.e. $f(S t)$ satisfies Equation 11). The process defining entity $C$ with a sensitive to predicate $S(S t)$ in any state $r$ is:

$$
C_{r}=r: \Psi \rightarrow C_{f(r)}
$$


where $\Psi$ is the set of states that satisfy $C$ 's activation condition: $\Psi=\left\{t: T_{C} \mid\right.$ $S(t)\}$.

The traces of the process defined by a VSPEC entity is a sequence of abstract states the entity enters. When the abstract state changes to an abstract state that satisfies the entity's activation condition ( $r$ in Equation 2), the transformation defined by the requires and ensures predicates $(f(S t))$ is applied to $r$. This generates a new abstract state and the entity behaves like the process defined by $C_{f(r)}$. The abstract states that satisfy $C$ 's activation condition form the alphabet of $C$. Thus, every trace of $C$ contains only elements from $\Psi$.

CSP's concurrency operator $(\|)$ combines component processes to define the behavior of a VSPEC architecture. Let $C_{1}, C_{2}, \ldots, C_{n}$ be the processes represented by Equation 2 for the set of VSPEC component instances in architecture $\mathcal{A}$. The process representing architecture $\mathcal{A}$ is:

$$
\mathcal{A}=C_{1}\left\|C_{2}\right\| \ldots \| C_{n}
$$

When a state change occurs that satisfies some component's activation condition, the component performs its specified transformation to its abstract state. This change is propagated to the concrete state of the architecture where the activation condition of another component may be satisfied. This causes the process to repeat until the system changes to a concrete state where no component's activation condition is satisfied. The system then waits until some external device changes the concrete state to one that activates some component in the architecture to start the process again.

In the CSP model of a VSPEC process, this notion can be understood by examining the possible traces of $\mathcal{A}$ from Equation 3 Hoare [12] defines traces over parallel composition, traces $\left(C_{1} \| C_{2}\right)$, as:

$$
\left\{t \mid\left(t \uparrow \alpha C_{1}\right) \in \operatorname{traces}\left(C_{1}\right) \wedge\left(t \uparrow \alpha C_{2}\right) \in \operatorname{traces}\left(C_{2}\right) \wedge t \in\left(\alpha C_{1} \cup \alpha C_{2}\right)^{*}\right\}
$$

Recall that in CSP [12, $t \uparrow \alpha P$ restricts the trace $t$ to contain only events that appear in the alphabet of $P$. Thus, the traces of a parallel composition of components are all traces that when restricted to the alphabet of each component yield a trace of that component. Furthermore, traces of $C_{1} \| C_{2}$ only contain events from the alphabet of the two components. This means that every trace of $\mathcal{A}$ contains only elements that satisfy the activation condition of at least one component in $\mathcal{A}$.

\section{Generating Semantic Models}

VSPEC semantics are used to verify requirements by transforming VSPEC into Larch Shared Language equivalent representations and using the Larch Prover. The SAVANT VHDL parser [15] has been extended to recognize VSPEC components and generate LSL representations. To support translation, background theories 
representing: (i) VHDL data types; (ii) the store model; and (iii) the CSP semantics were implemented to provide underlying semantics. The resulting LSL models are suitable for various verification activities using the Larch Prover.

Extending the SAVANT parser to recognize VSPEC and generate LSL required extending the language recognizer and the AIRE intermediate form. The parser was extended in the canonical fashion to recognize and type check VSPEC components. To generate LSL representations, the AIRE intermediate form has been extended to include: (i) VSPEC constructs; and (ii) "publish" methods to generate LSL traits representing components and architectures.

Generating LSL representations for VHDL data types, the store model and CSP functions involves defining LSL theories for each construct. For each VHDL type, a parameterized trait was developed. When the associated type is used in a specification, the appropriate trait is included to provide a theory for that type in the LSL model. Similar techniques are used for the store and CSP models. LSL traits defined by Chalin [8] were reused here after some modification and reverification. For CSP functions, the CSP axiomatization developed by Camilleri [16] is adapted. Camilleri's original theories were developed in HOL [17, thus requiring translation to LSL and verification.

The only challenging task associated with translating Camilleri's CSP axiomatization involved moving from a higher order logic representation to a first order representation. Specifically, the CSP choice operation plays a major role in the VSPEC definition. In the HOL axiomatization, choice is parameterized over a choice operation. In LSL, this is achieved using a parameterized trait with the choice function as a parameter. The instantiation of the trait parameter is achieved at parse time requiring a new version of the choice trait to be instantiated for each instance of choice. The higher order representation allows instantiation of the choice function at proof time making for dramatically simpler proof activities.

Each VSPEC component is translated into an associated LSL trait. Brevity prevents the expansion of a component in this paper, however these traits share a common structure centering on: (i) the pre- and post-conditions; and (ii) the CSP choice operator. Specifically, the choice operator is specialized by specifying a choice function and an alphabet. The alphabet specifies what states can occur next and was defined as $\Psi$ earlier. The choice function generates the next state and is defined using the pre- and post-condition of the component.

Figure 5 shows the template used to generate LSL traits for each system component. The trait is instantiated using the table shown in Table 1 In the template, operations $I$ (pre) and $O$ (pre,post) are instantiated with the VSPEC component's requires and ensures clauses respectively. The sensitive to clause is incorporated in the alphabet of the CSP process associated with each component. Recall that the alphabet of the component is defined to be the set of states satisfying the activation condition. Note that the constrained by clause is not involved in the definition. Performance constraints are evaluated using PDL as described in a later section. 


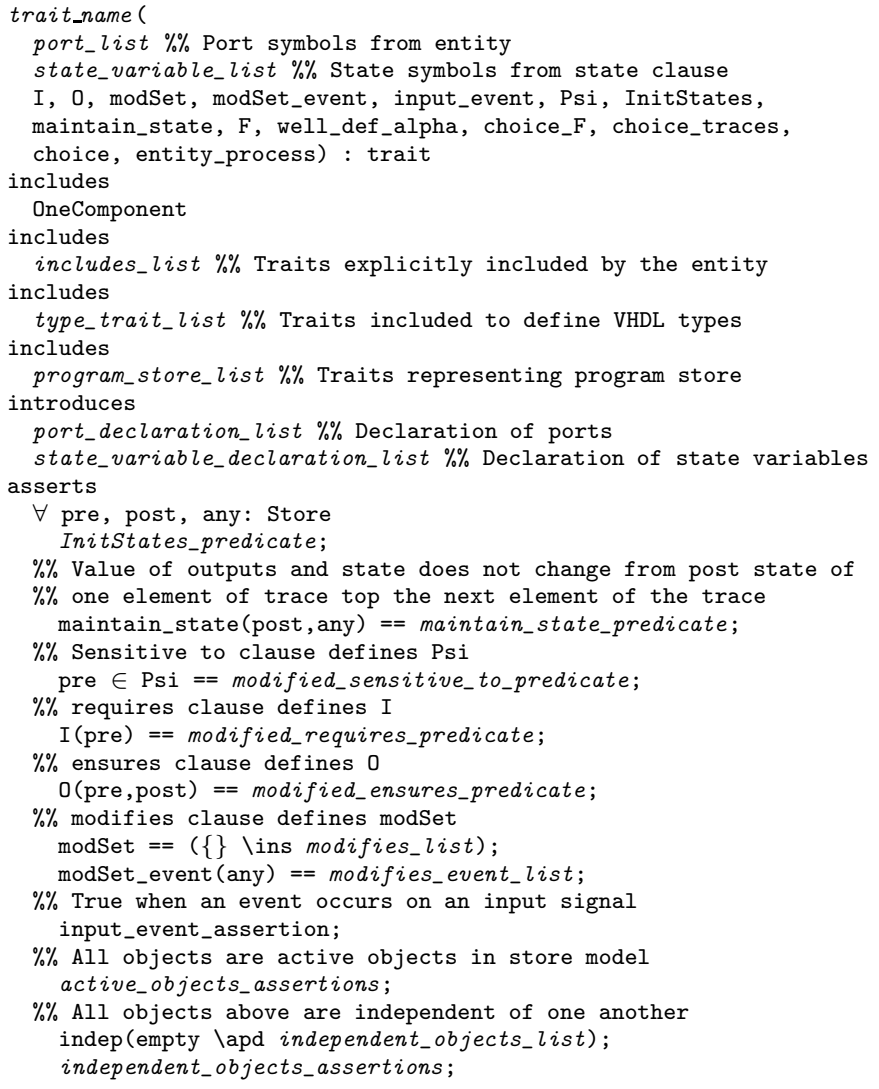

Fig. 5. Template specialized to generate traits for single components.

\section{Verification}

Correctness checks in VSPEC take three fundamental forms: (i) component verification; (ii) interconnection verification; and (iii) bisimulation. Component and interconnection verification represent partial correctness checks used to quickly assess the quality of a specification. Bisimulation precisely defines the relationship between a VSPEC architecture and an associated system specification. Although component and interconnection verification only verify specific system properties, they are frequently simpler to prove than bisimulation relationships.

Component verification allows users to specify properties of a single component using LSL. The component is transformed into an LSL trait and the Larch Prover called with the property as a theorem. Component interconnections are not considered and typically only the precondition, postcondition and activation condition are included the verification. This is the simplest verification activity and is used simply to verify properties of single VSPEC components. 


\begin{tabular}{|c|c|}
\hline Name from Template & Description \\
\hline trait_name & entity_name_trait \\
\hline port_list & Comma-separated list of all entity port names. \\
\hline state_variable_list & Comma-separated list of all entity state variable names. \\
\hline includes_list & $\begin{array}{l}\text { Comma-separated list of names of all traits explicitly } \\
\text { included in the entity. }\end{array}$ \\
\hline type_trait_list & $\begin{array}{l}\text { Comma-separated list of trait names that define the VHDL } \\
\text { types needed in this entity. }\end{array}$ \\
\hline program_store_list & $\begin{array}{l}\text { Comma-separated list of sorted projections of the program } \\
\text { store (SProjStore) needed in this trait. }\end{array}$ \\
\hline port_declaration_list & LSL constant declaration for each port signal in the entity. \\
\hline state_variable_declaration_list & LSL constant declaration for each state variable in the entity. \\
\hline InitStates_predicate & $\begin{array}{l}\text { Predicate to define initial Stores an entity can enter. Takes into } \\
\text { account the initial value of all entity state variables. }\end{array}$ \\
\hline maintain_state_predicate & $\begin{array}{l}\text { Assertion that the value of all outputs and state variables } \\
\text { does not change from Store post to Store any. }\end{array}$ \\
\hline modified_sensitive_to_predicate & $\begin{array}{l}\text { Entity's sensitive to clause predicate converted to LSL } \\
\text { format with all port and state variable references replaced with } \\
\text { call to Chalin's val operator for the proper Store (pre or post). }\end{array}$ \\
\hline modified_requires_predicate & $\begin{array}{l}\text { Entity's requires clause predicated converted to LSL format } \\
\text { with all port and state variable references replaced with call to } \\
\text { Chalin's val operator for the proper Store (pre or post). }\end{array}$ \\
\hline modified_ensures_predicate & $\begin{array}{l}\text { Entity's ensures clause predicated converted to LSL format } \\
\text { with all port and state variable references replaced with call to } \\
\text { Chalin's val operator for the proper Store (pre or post). }\end{array}$ \\
\hline modifies_list & $\begin{array}{l}\text { List of all modified port signals and state variables (including } \\
\text { "implicitly" modified ones). The \ins operator separates each } \\
\text { element in this list. }\end{array}$ \\
\hline modifies_event_list & $\begin{array}{l}\text { Assertion there is an event on the value of at least one } \\
\text { modified object in Store any. }\end{array}$ \\
\hline input_event_assertion & $\begin{array}{l}\text { Disjunction of statements that an event occurred on an input } \\
\text { signal in state any }\end{array}$ \\
\hline active_objects_assertions & $\begin{array}{l}\text { Assertion of the form } X \backslash \text { in activeObjs (any) for every } \\
\text { object X (i.e. each signal and state variable) in the entity. }\end{array}$ \\
\hline independent_objects_list & $\begin{array}{l}\text { List of all objects in the entity. The \apd operator } \\
\text { separates each element in this list. }\end{array}$ \\
\hline independent_objects_assertions & $\begin{array}{l}\text { One commented out assertion of the form } \\
\text { depOn }(d w n(x) \text {, dwn }(y)) \text { for every possible combination of } \\
\text { port signals and state variables } x \text { and } y \text {. }\end{array}$ \\
\hline
\end{tabular}

Table 1. Summary of substitutions to create a trait for an associate entity using the entity template.

Interconnection verification examines specific relationships between components. As the name implies, interconnection verification centers primarily on properties of interconnected ports. In most cases, users select from pre-defined conditions. Proof obligations are generated and verified automatically in most cases. Specific examples of interconnection verifications include proving: (i) all outputs from a component are legal inputs to another; (ii) some output from a component will activate another; and (iii) all inputs activating a component are legal component inputs. Many similar, additional obligations can be generated and discharged automatically.

Bisimulation allows a user to verify that an abstract architecture correctly implements its requirements. This verification obligation is generated automatically by the VSPEC parser and is based on weak bisimulation [18]. A weak bisimulation (or simply bisimulation) condition holds when a sequence of states 
in the architecture (or concrete) model produces the desired single state change specified by the system level (or abstract) model (see Figure 66). Only the first and last state of the concrete state sequence are significant. The specific state sequence leading from the initial concrete state to the final concrete state is ignored.

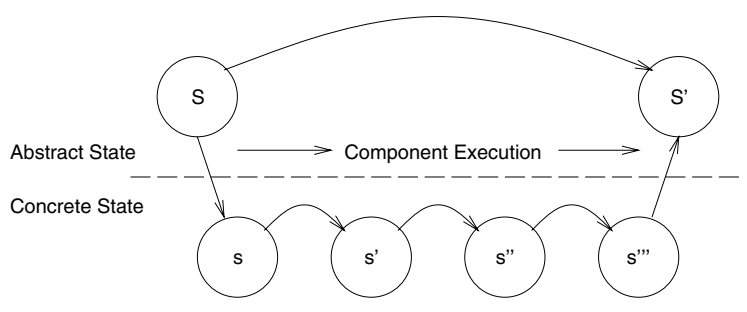

Fig. 6. Concrete state changes associated with a single abstract state change.

Equation 5 is a weak bisimulation correctness obligation for showing abstract architecture $\mathcal{A}$ satisfies a single abstract state change. Here, $\Psi_{\mathcal{A}}$ is the set of concrete states where the activation condition of at least one component in $\mathcal{A}$ is true. The obligation states that for all traces of the concrete state starting in a state whose abstract projection satisfies the abstract specification's pre-condition, either the resulting process state satisfies the component postcondition or the process can consume the state and continue.

$$
\forall \tau: \operatorname{traces}(\mathcal{A}) \cdot I\left(\operatorname{abs}\left(\tau_{0}\right)\right) \wedge \mathcal{A} / \tau=\mathcal{A}_{s} \Rightarrow\left(O\left(\operatorname{abs}\left(\tau_{0}\right), a b s(s)\right) \vee s \in \Psi_{\mathcal{A}}\right)
$$

In addition to functional verification, the current VSPEC tool suite supports constraint verification using The Performance Description Language (PDL) 19]. The semantics of constraints is defined using LSL in a fashion similar to the other VSPEC clauses. However, constraint evaluation requires significant manipulation of intervals. This activity proved to be exceptionally difficult in the Larch toolset.

PDL is a language designed explicitly for representing and evaluating constraint information. Specifically, PDL provides a mechanism for representing and evaluating interval mathematics representations. In PDL, designers define models for various constraint types. These models are then used to instantiate architectures of components. Each interconnected component is assigned one model for each constraint to be evaluated.

VSPEC constraint evaluation allows the user compare a systems level constraint description to an architectural description. For each VSPEC constraint, a PDL model has been defined. Each model minimally defines the data type associated with the constraint, and a function to combine two constraint values. An architecture is derived from the VHDL architecture and populated with the 
various constraint models. Using the PDL evaluator and the combine operators associated with each constraint, a system level constraint value is calculated. This value is then compared with the system level specification to determine if the architecture meets its associated systems level performance requirements.

\section{$6 \quad$ VSPEC in Practice}

VSPEC has been evaluated in both academic and industrial settings. Many example specifications have been written in the research setting. Two of which, the Move Machine and Find, represent significant milestones in the VSPEC lifecyle. More recently, TRW has used VSPEC to specify two significant avionics components.

\subsection{The Move Machine}

The first significant specification developed using VSPEC was done by Baraona [4. The Move Machine is a simple CPU specification where operations are mapped to memory locations. The only operations performed by the Move Machine move data from one memory location to another. The Move Machine was specified as an instruction interpreter and at the register transfer level. Specifications were written, however no analysis was performed. The Move Machine exercise served to shake out initial problems in the VSPEC semantics. Although no verification was performed, the exercise represented the first actual vSPEC usage example. Furthermore, the example represents a common class of systems and provided an excellent usage example for early users.

\subsection{Find}

The Find system and architecture specifications presented in Figure 2 represent the first complete bisimulation verification activity performed using VSPEC. Like the Move Machine, Find was developed as a synthesis benchmark and exhibits some interesting modeling capabilities. Specifically, the use of activation conditions to model data caching. The Find architecture decomposes the problem into two components: (i) a sorting component; and (ii) a binary search component. The architecture is specified to cache the results of sorting so that if the input array does not change, it is not resorted. The result is a more efficient search.

Specifications similar to those presented in Figure 2 were written and parsed by the original VSPEC parser. Formal models were generated by the parser and the Larch Prover was used to perform the verification. For this example, the proof obligations necessary to show the bisimulation relationship were written by hand. Although the verification was completed successfully, this activity demonstrated limitations in using LSL and the Larch Prover for verifications of this size. The fundamental problem was the first order nature of LSL and the mechanisms used to specify the basic CSP theories. 


\subsection{Pulse Interval Processor}

In an initial technology transfer effort, VSPEC was used jointly by TRW and The University of Cincinnati to model the Pulse Interval Processing (PIP) section of an Interrogate Friend or Foe (IFF) transponder. The IFF transponder redesign represents a real Digital Signal Processing (DSP) product being designed and fielded by TRW. Although VSPEC was not used in the actual design flow, we were given access to specifications and worked with engineers designing the system. Unfortunately the PIP architecture is proprietary to TRW and details of the specification cannot be presented here. However, several important results are presented.

The PIP is an interesting challenge problem because of its means of encoding information. The PIP's function is to: (i) receive pulses from a digital signal processing system; (ii) transform pulse trains into commands; and (ii) produce appropriate output in response to those commands. Commands are used by other aircraft and air traffic control systems to gather information about an aircraft's altitude, position, mission and origin. The PIP is a particularly interesting model because information is encoded in the time difference between received pulses. This presented an interesting specification challenge as VSPEC does not use a temporal logic.

The specification was written using LSL to represent pulse times and their associated receive times. Pulse streams are processed by examining each pulse with respect to the PIP state and receive time of the last pulse. When a pulse is received its receive time is stored in the PIP state. When a second pulse is received, the difference between its receive time and the stored receive time determines the command issued. The VSPEC state clause is used to maintain the last pulse received and a nested IF-THEN-ELSE statement is used to represent various processing cases.

The PIP specification was partially verified by automatically generating interconnect and component proof obligations. We were able to verify several interconnect conditions and TRW found one type error that had not been caught in the actual design flow. This error was not significant and would likely have been caught during design inspection or testing. No bisimulation relationships were specified or verified as the PIP architecture was established before beginning this specification task. As an extensive design activity for PIP preceded our efforts and this represented a redesign of a well understood system, the use of VSPEC did not seriously impact the actual design flow. However, design engineers commented that writing VSPEC made specification of test vectors much simpler. This represents an interesting and somewhat unexpected result.

\subsection{Real Time Monitor}

The Real Time Monitor (RTM) is a proprietary special purpose signal processor used in military aircraft avionics subsystems. It is similar to the PIP except it contains programmability features allowing it to process multiple pulse streams and recognize multiple protocols. The RTM processor was specified by TRW 
exclusively with only VSPEC language assistance from the developers. Little verification was performed due to time restraints on the overall project.

Although details of the activity are considered proprietary, TRW acknowledges that using VSPEC played a key role in reducing estimated product costs by $25 \%$. They observed that although VSPEC increased cost and development time during requirements analysis and representation, early mitigation of errors, precise requirements capture and ease of test vector generation compensated for initial costs. Specifically, they reported that the result of the VSPEC specification activity resulted in much more precise and complete specifications. Even without extensive verification, they reported discovering and mitigating ambiguities in the specifications. Although only one legitimate error was discovered, using VSPEC forced them to be more precise in their specification activities. However, it should be noted that the primary motivation for writing VSPEC was a promise for automated verification, not more precise specifications.

An unexpected result was TRW's engineer's belief that having VSPEC specifications made defining and generating test vectors an easier task. In the design and implementation of avionics systems such as those developed by TRW, test vector generation and maintenance is a resource intensive task. TRW engineers reported that having VSPEC specifications made identification of test cases and definition of test vectors easier. Although unexpected, careful analysis indicates this result is not surprising. It should be the case that test vectors correspond with requirements and ultimately that is what they are designed to test. This result is being explored in a follow on effort to determine if VSPEC can systematically impact the testing process.

TRW commented further that mature tools integrated into VHDL design environments would contribute to potential cost savings. Although this represents a development task, it does indicate the importance of integrating formal methods into traditional design flows.

\subsection{Evaluation Summary}

The VsPEC language and tools were evaluated with respect to two internal and two industrial examples. Internal examples were evaluated by students at The University of Cincinnati. The first industrial example was evaluated jointly with TRW and the second evaluated solely by TRW. Table 2 represents specification and verification operations performed in each example. Each evaluation was completed to a large degree of success. Although language semantics and tool problems were certainly found, TRW is continuing to actively use VSPEC and continues to support tool development activities.

\section{Related Work}

Odyssey Research Associates (ORA) has developed Larch/VHDL, an alternative Larch interface language for VHDL [20]. Larch/VHDL is targeted for formal analysis of a VHDL description and ORA is defining a formal semantics for VHDL 


\begin{tabular}{|c|c|c|c|c|}
\hline$\overline{\text { Example }}$ & $\begin{array}{l}\text { System } \\
\text { Spec }\end{array}$ & $\begin{array}{c}\text { Architecture } \\
\text { Spec }\end{array}$ & $\begin{array}{c}\text { Interconnect } \\
\text { Verification }\end{array}$ & $\begin{array}{c}\text { Bisimulation } \\
\text { Verification }\end{array}$ \\
\hline Move Machine & $\mathrm{X}$ & $\mathrm{X}$ & & \\
\hline Find & $\mathrm{X}$ & $\mathrm{X}$ & $\mathrm{X}$ & $\mathrm{X}$ \\
\hline PIP & & $\mathrm{X}$ & $\mathrm{X}$ & \\
\hline RTM & & $\mathrm{X}$ & & \\
\hline
\end{tabular}

Table 2. Major vsPEC evaluation activities.

using LSL. The LSL representations are used in a traditional theorem prover to verify system correctness. Larch/VHDL annotations are added to a specific VHDL description to represent proof obligations for the verification process. This differs from VSPEC's purpose of representing requirements and design decisions at high levels of abstraction.

Augustin et al.'s VAL [21] is another attempt to annotate VHDL. The purpose of a VAL annotation to a VHDL description is to document the design for verification. VAL provides mechanisms for mapping a behavioral description to a structural description. Two VAL/VHDL descriptions of a design can be transformed into a self-checking VHDL program that is simulated to verify that the two descriptions implement the same function. This is once again slightly different than VSPEC's purpose of high level requirements representation.

The abstract architecture representation capabilities of VSPEC are also fairly closely related to several architecture description languages that have been developed to describe software architectures 22. Some of the more well known architecture description are UniCon [23, WRIGHT [24, 25] and RAPIDE [26, 27]. Each of these languages allow the definition of components and connectors to define a software architecture. This is very similar to the VHDL notion of a structural architecture.

Allen and Garlan's WRIGHT language is of particular interest when discussing VSPEC because a WRIGHT component is defined with a variant of CSP. Unlike VSPEC's use of CSP to define component synchronization, WRIGHT uses CSP to define component behavior as well. A WRIGHT description consists of a collection of components interacting via instances of connector types. WRIGHT's CSP descriptions define the sequence of events a component or connector participates in.

\section{Conclusions and Current Status}

Several problems and complexities of the Larch-based semantics were discovered in our prototyping activities. In an effort to eliminate difficulties, the VSPEC semantics have been transformed into PVS 28 form. The use of higher order logic substantially simplifies the specification and verification of CSP properties. The VSPEC parser has been re-implemented in Java and generates PVS models 
in place of Larch. The higher order nature of PVS and maturity of its associate toolset will dramatically improve verification efficiency.

VSPEC is currently involved in several commercialization efforts. First, under Air Force and TRW sponsorship, the vSPEC toolset is being integrated with Mentor Graphics' Renoir development environment. This integration will allow VSPEC to be evaluated by engineers in their traditional design cycle. Second, under DARPA and EDAptive Computing sponsorship, VSPEC is being used to facilitate component retrieval by comparing component requirements with available components described by VSPEC. Comparing requirements with components formally supports high assurance in the quality of the match. Finally, under TRW sponsorship we have begun an effort to generate test vectors from VSPEC specifications.

This paper briefly presented the VSPEC language and its associated semantics. The semantics of single components were defined using a canonical axiomatic approach. Activation conditions were described and a CSP-base semantics provided. The use of VHDL architectures to describe abstract architectures was then discussed. The paper concluded with a presentation of examples used to evaluate the system and results of those activities.

\section{References}

[1] IEEE Standard VHDL Language Reference Manual. New York, NY, 1993.

[2] P. Alexander, P. Baraona, and J. Penix. Using Declarative Specifications and Case-Based Planning for System Synthesis. Concurrent Engineering: Research and Applications, 2(4), 1994.

[3] P. Alexander, P. Baraona, and J. Penix. Application of Software Synthesis Techniques to Composite Systems. In Computers in Engineering Symposisum of the ASME ETCE, Houston, TX, January 1995.

[4] P. Baraona, J. Penix, and P. Alexander. VSPEC: A Declarative Requirements Specification Language for VHDL. In Jean-Michel Berge, Oz Levia, and Jacques Rouillard, editors, High-Level System Modeling: Specification Languages, volume 3 of Current Issues in Electronic Modeling, chapter 3, pages 51-75. Kluwer Academic Publishers, Boston, MA, 1995.

[5] Phillip Baraona and Perry Alexander. Abstract architecture representation using VSPEC. VLSI Design, 9(2):181-201, 1999.

[6] J. Guttag, J. Horning, and J. Wing. The Larch Family of Specification Languages. IEEE Software, 2(5):24-36, 1985.

[7] John V. Guttag and James J. Horning. Larch: Languages and Tools for Formal Specification. Springer-Verlag, New York, NY, 1993.

[8] Patrice Chalin. On the Language Design and Semantic Foundation of LCL, a Larch/C Interface Specification Language. PhD thesis, Concordia University, Department of Computer Science, Montreal, Quebec, Canada, December 1995.

[9] John V. Guttag and James J. Horning. Introduction to LCL, A Larch/C Interface Language. Technical Report 74, Digital Equipment Corporation Systems Research Center, 130 Lytton Avenue, Palo Alto, CA 94301, July 1991.

[10] Gary T. Leavens. Larch/C++ Reference Manual. Available at: ftp://ftp.cs.iastate.edu/pub/larchc++/lcpp.ps.gz, 1995. 
[11] David Guaspari, Carla Marceau, and Wolfgang Polak. Formal Verification of Ada Programs. IEEE Transactions on Software Engineering, 16(9):1058-1075, September 1990.

[12] C. A. R. Hoare. Communicating Sequential Processes. Prentice-Hall, Englewood Cliffs, 1985.

[13] Knowledge Based Software Engineering Laboratory, University of Cincinnati. VSPEC Language Reference Manual, 1996.

[14] R.D. Tennent. Principles of Programming Languages. Computer Science Series. Prentice-Hall International, 1981.

[15] P. A. Wilsey, D. E. Martin, and K. Subramani. Savant/tyvis/warped: Components for the analysis and simulation of vhdl. In VHDL Users' Group Spring 1998 Conference, pages 195-201, 1998.

[16] Albert John Camilleri. Mechanizing CSP trace theory in higher order logic. IEEE Transactions on Software Engineering, 16(9):993-1004, September 1990.

[17] M. J. C. Gordon. HOL: A proof generating system for higher-order logic. In G. Birtwistle and P. A. Subrahmanyam, editors, Current Trends in Hardware Verification and Automated Theorem Proving, pages 73-128. Springer-Verlag, 1989.

[18] Robin Milner. A Calculus of Communicating Systems, volume 92 of Lecture Notes in Computer Science. Springer-Verlag, Berlin, 1980.

[19] Ranga Vemuri, Ram Mandayam, and Vijay Meduri. Performance modeling using PDL. Computer, 29(4):44-53, April 1996.

[20] D. Jamsek and M. Bickford. Formal Verification of VHDL Models. Technical Report RL-TR-94-3, Rome Laboratory, Griffiss Air Force Base, NY, March 1994.

[21] L. Augustin, D. Luckham, B. Gennart, Y. Huh, and A. Stanculescu. Hardware Design and Simulation in VAL/VHDL. Kluwer Academic Publishers, Boston, MA, 1991.

[22] D. Garlan and M. Shaw. An Introduction to Software Architecture. In V. Ambriola and G. Tortora, editors, Advances in Software Eng. and Knowledge Eng., volume 2, pages 1-39. World Scientific, New York, 1993.

[23] M. Shaw, R. DeLine, D. Klein, T. Ross, D. Young, and G. Zelesnik. Abstractions for Software Architecture and Tools to Support Them. IEEE Transactions on Software Engineering, 21(4):314-335, April 1995.

[24] R. Allen and D. Garlan. Formalizing Architectural Connection. In Proc. Sixteenth International Conference on Software Engineering, pages 71-80, May 1994.

[25] R. Allen and D. Garlan. A Case Study in Architectural Modelling: The AEGIS System. In Proceedings of the 8th International Workshop on Software Specification and Design, March 1996.

[26] D. Luckham, J. Kenney, L. Augustin, J. Vera, D. Bryan, and W. Mann. Specification and Analysis of System Architecture Using Rapide. IEEE Transactions on Software Engineering, 21(4):315-355, April 1995.

[27] D. Luckham and J. Vera. An Event-Based Architecture Definition Language. IEEE Transactions on Software Engineering, 21(9):717-734, September 1995.

[28] Judy Crow, John Rushby, Natarajan Shankar, and Mandayan Srivas. A Tutorial Introduction to PVS. SRI International, Menlo Park, CA, June 1995. Presented at WIFT'95. 